Conclusion Incidence of chest pain presenting to our ED was $1 \%$. The commonest recorded cause was musculoskeletal. Fewer than $1 \%$ had a possible cardiac aetiology for chest pain. ECG is a useful test for children presenting with chest pain. Very few patients with mild ST elevation had cardiac enzyme levels checked.

\section{G168(P) ANTENATAL MANAGEMENT OF FETAL CARDIAC DISEASES, A SINGLE CENTRE EXPERIENCE IN EGYPT}

H ElMarsafawy, S Rakha, R El Ashry, Y Al Tonbary. Department of Pediatrics, Faculty of Medicine, Mansoura University, Mansoura, Egypt

\subsection{6/archdischild-2015-308599.163}

Aims The antenatal diagnosis of congenital heart diseases (CHD) is crucial for fetal and perinatal management; however, there is little in literature regarding the pharmacologic intervention for fetal CHD in Egypt. Therefore, we attempted to detect fetal cardiac structural or functional abnormalities using fetal echocardiography and plan fetal pharmacologic intervention without endangering the mother's life.

Methods Cases fulfilled inclusion criteria were diagnosed using detailed fetal Echocardiographic examinations and antenatal treatment was described if indicated after a written consent. Cardiovascular profile score (CVPS) was used to assess the response to treatment. Postnatal transthoracic Echo was done to confirm diagnosis with follow up till the end of neonatal period to determine outcome.

Results Fetuses fulfilled inclusion criteria were 143 with a mean gestational age at diagnosis $27.59 \pm 5.41$ weeks, mean maternal age at diagnosis was $26.64 \pm 5.428$ years, the most frequent cause of referral was family history of CHD (34.1\%). Twenty fetuses (14\%) received antenatal therapy. Fetuses with heart failure due to structural cardiac defects $(n=4)$ and functional non arrhythmic heart failure $(n=8)$ received digoxin while cases with fetal tachyarrhythmia $(n=6)$ received digoxin and/or sotalol or flecainide and fetuses with immune mediated fetal heart block $(n=2)$ received dexamethazone. Success in tachyarrhythmia was statistically significant regarding CVPS $(p=0.038)$ and heart rate changes $(p=0.002)$ but statistically insignificant regarding CVPS in structural defects $(p=0.102)$ and nonarrhythmic functional heart failure $(p=0.343)$.

Conclusion Antenatal cardiac pharmacologic intervention is possible with hydrops fetalis reversal in fetal tachyarrhythmia and resolution of first degree immune mediated atrioventricular block. On the contrary, no response to antenatal digoxin use in fetal structural heart failure and limited response in non-arrhythmic functional heart failure.

\section{G169(P) EXPLORING ETHNIC VARIATION IN INFANTS WITH CONGENITAL HEART DEFECTS UNDERGOING PAEDIATRIC CARDIAC SURGERY}

\footnotetext{
${ }^{1} \mathrm{RL}$ Knowles, ${ }^{1} \mathrm{D}$ Ridout, ${ }^{2} \mathrm{~S}$ Crowe, ${ }^{3} \mathrm{~J}$ Tregay, ${ }^{3} \mathrm{~J}$ Wray, ${ }^{4} \mathrm{D}$ Barron, ${ }^{5} \mathrm{D}$ Cunningham, ${ }^{6} \mathrm{R}$ Parslow, ${ }^{7} \mathrm{R}$ Franklin, ${ }^{3} \mathrm{C}$ Bull, ${ }^{3} \mathrm{~K}$ Brown. ${ }^{1}$ Institute of Child Health, University College London, London, UK; ${ }^{2}$ Clinical Operational Research Unit, University College London, London, UK; ${ }^{3}$ Great Ormond Street Hospital NHS Foundation Trust, London, UK; ${ }^{4}$ Birmingham Children's Hospital NHS Foundation Trust, Birmingham, UK; ${ }^{5}$ National Institute for Cardiovascular Outcomes Research, University College London, London, UK; ${ }^{6}$ Paediatric Intensive Care Audit Network, University of Leeds, Leeds, UK; ${ }^{7}$ Royal Brompton and Harefield Hospitals NHS Foundation Trust, London, UK
}

Introduction North American researchers have reported ethnic differences in the prevalence and short-term outcomes of congenital heart defects (CHDs), which may reflect genetic variation, environmental exposures or healthcare access. It is unclear whether ethnic differences in CHD frequency and outcomes also exist in the UK population and healthcare system.

Aim To examine national paediatric cardiac surgical audit data for ethnic differences in the frequency of different CHD subtypes, associated comorbidities and short-term outcomes for infants operated in the first year of life.

Methods Individual records from the national congenital cardiac surgical audit (NICOR) of UK infants aged under 12 months who had a cardiac surgery or intervention between 01/01/2005 and 31/12/2010, were matched with intensive care admission records in the Paediatric Intensive Care Audit Network (PICANET) and linked records were obtained for 8481 (86\%) of operated infants. Census-derived categories for ethnic classification were used.

Results Children who were operated within the first year of life for major CHD represented 2.2 (95\% Confidence Intervals [CI] 2.2, 2.3) per 1000 live births. Compared with children of white ethnicity, children of Asian ethnicity were more likely to have cardiac surgery $(2.3[2.3,2.4]$ and $3.2[3.0,3.5]$ per 1000 live births respectively) in the first year of life. CHD subtypes that were significantly over-represented within the Asian ethnic group included single ventricle (SV), transposition of the great arteries, pulmonary atresia, tetralogy of Fallot (TOF) and septal defects; in the Black ethnic group, atrioventricular septal defect and SV were over-represented, while TOF and aortic stenosis were under-represented. Preterm birth occurred in 14\% of babies, almost twice the general population rate, and associated non-cardiac anomalies were reported in $21 \%$ of affected infants, however no significant ethnic variation was observed. There were 246 deaths during the first year after hospital discharge following surgery but no significant ethnic differences in short-term mortality were identified.

Conclusion The risk of CHD intervention in infants aged under one year varied by ethnic group, and children of Asian ethnicity were at greater risk. No ethnic differences in short-term post-discharge mortality were identified, however longer-term outcomes should be explored.

\section{G170(P) IMPROVING ANTENATAL DETECTION RATES OF SIGNIFICANT CONGENITAL CARDIAC LESIONS IN A DISTRICT GENERAL HOSPITAL}

H Aughey, L Guilder, Y Kumar. Paediatric Department, Royal Cornwall Hospital Trust, Truro, UK

\subsection{6/archdischild-2015-308599.165}

Introduction Congenital heart disease (CHD) affects around 1\% of pregnancies in the UK each year. Around half of these are major cardiac lesions requiring surgery or intervention within the $1^{\text {st }}$ year of life. Studies show that if CHD is detected before birth, there are significant benefits for babies, their families and for medical services around the time of birth and in the first year of life. Prenatal diagnosis and appropriate treatment may prevent the devastating consequences of early circulatory collapse, such as death and ischaemic brain damage.

Background An audit previously conducted in our district general hospital found that the antenatal detection rate of significant cardiac lesions in Jan 1998-Dec 1999 was 17.6\%. Following 